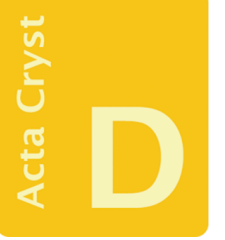

STRUCTURAL BIOLOGY

Volume 75 (2019)

Supporting information for article:

Structural basis for diversity of the mechanism of nucleotide hydrolysis by the aminoglycoside-2"-phosphotransferases

Clyde A. Smith, Marta Toth, Nichole K. Stewart, Lauren Maltz and Sergei B. Vakulenko 
Supplementary Information for

\section{Structural Basis for Diversity of the Mechanism of Nucleotide Hydrolysis by the Aminoglycoside-2" Phosphotransferases}

Clyde A. Smith ${ }^{\mathrm{a}, \mathrm{b}^{*}}$, Marta Toth $^{\mathrm{c}}$, Nichole K. Stewart ${ }^{\mathrm{c}}$, Lauren Maltz ${ }^{\mathrm{a}}$ and Sergei B. Vakulenko ${ }^{\mathrm{c} *}$

${ }^{a}$ Stanford Synchrotron Radiation Lightsource, SLAC National Accelerator Laboratory, Menlo Park, CA 94025 USA

${ }^{\mathrm{b}}$ Department of Chemistry, Stanford University, Stanford, CA 94305, USA

${ }^{\mathrm{c}}$ Department of Chemistry and Biochemistry, University of Notre Dame, Notre Dame, IN 46556, USA 

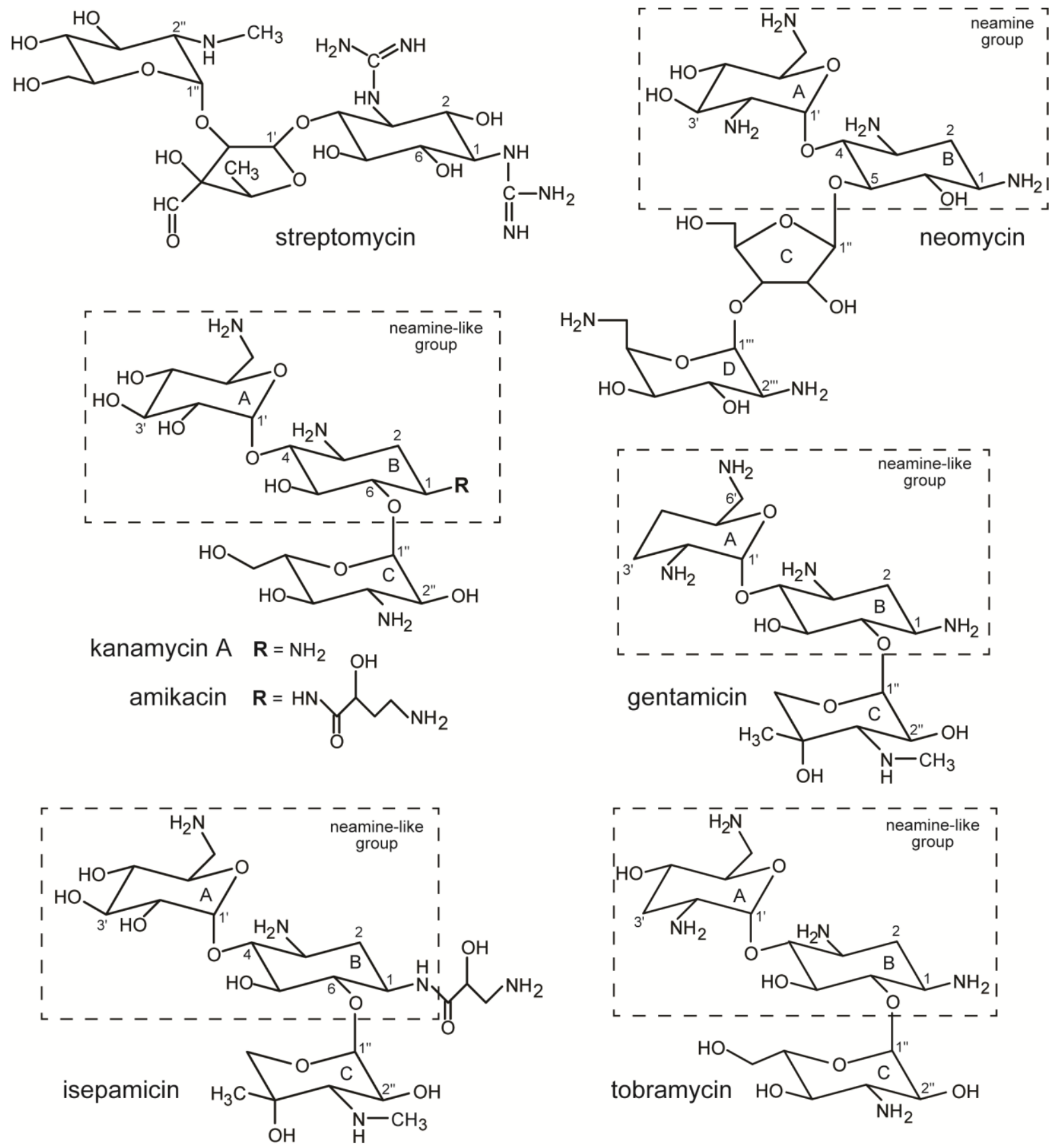

\section{Supplementary Figure S1}

Structures of some representative aminoglycoside antibiotics. Streptomycin is classified as an atypical aminoglycoside. Neomycin is an example of a 4,5-disubstitued aminoglycoside, and kanamycin, gentamicin, isepamicin and tobramycin are examples of 4,6-disubstituted aminoglycosides. Neamine, a degradation product of neomycin, comprises the A- and B-rings. The corresponding rings of the 4,6disubstituted aminoglycosides are designated as neamine-like groups, with one or two differences or deletions of side groups on the A-ring. 


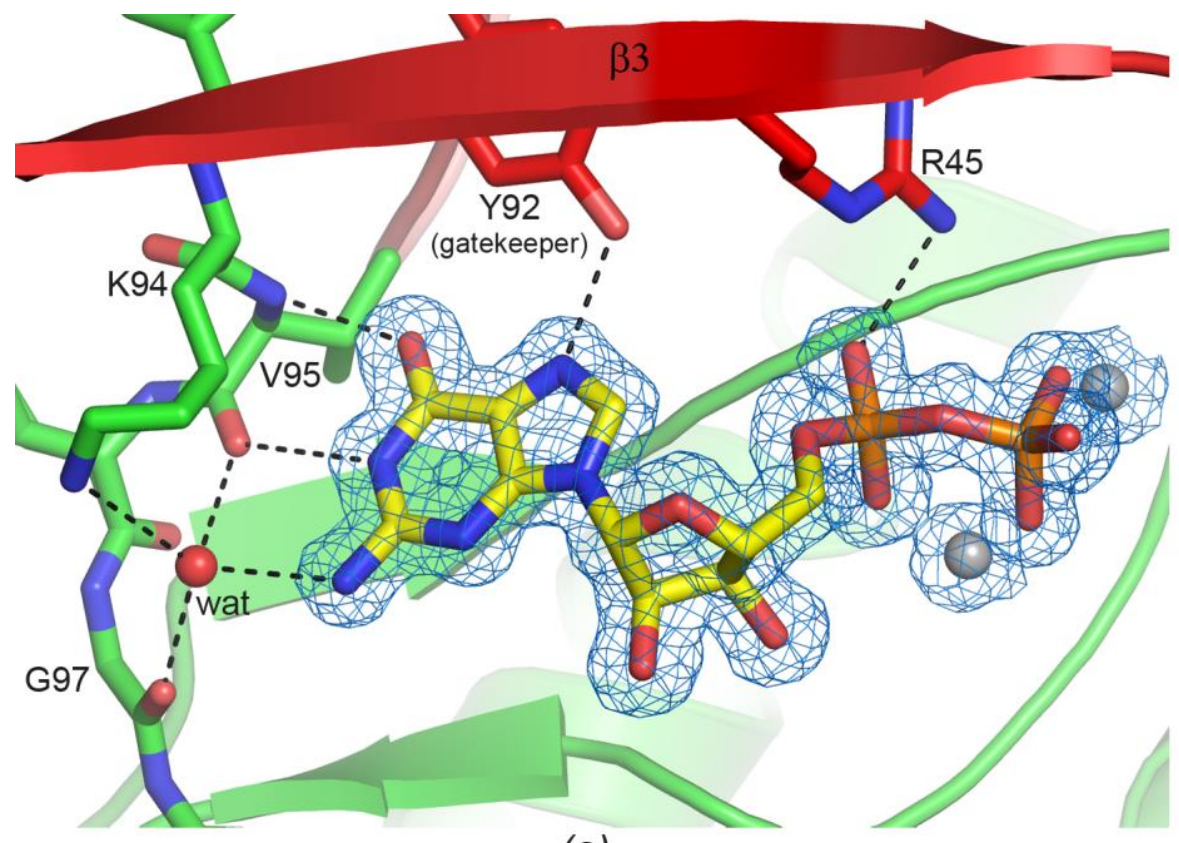

(a)

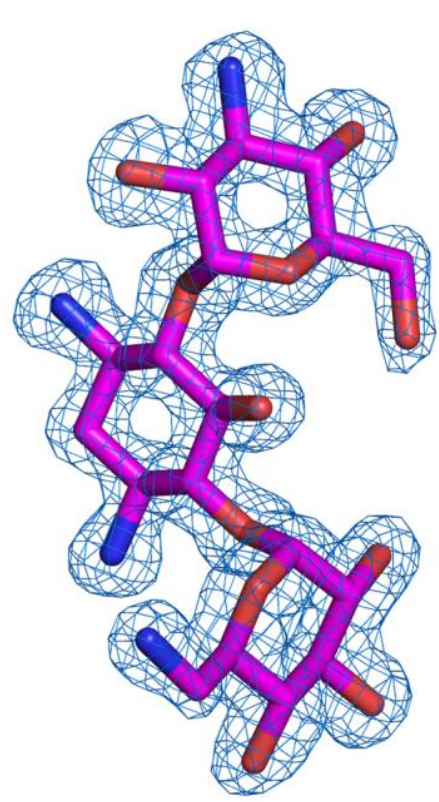

(b)

\section{Supplementary Figure S2}

Substrate and cofactor electron density. (a) The APH(2")-IIIa nucleotide binding site showing the final refined $2 F_{o}-F_{c}$ electron density map (blue mesh, $1.0 \sigma$ )) for the GDP (yellow sticks) and the two associated magnesium ions (gray spheres). The hydrogen bonding interactions between the protein and the GDP are indicated as dashed black lines. The coloring of the protein is similar to that in Fig. 1, where the N-terminal domain is red and the core sub-domain is green. $(b)$ The final refined $2 F_{o}-F_{c}$ electron density (blue mesh, 1.0 б) for the kanamycin (magenta sticks) in APH(2")-IIIa. 


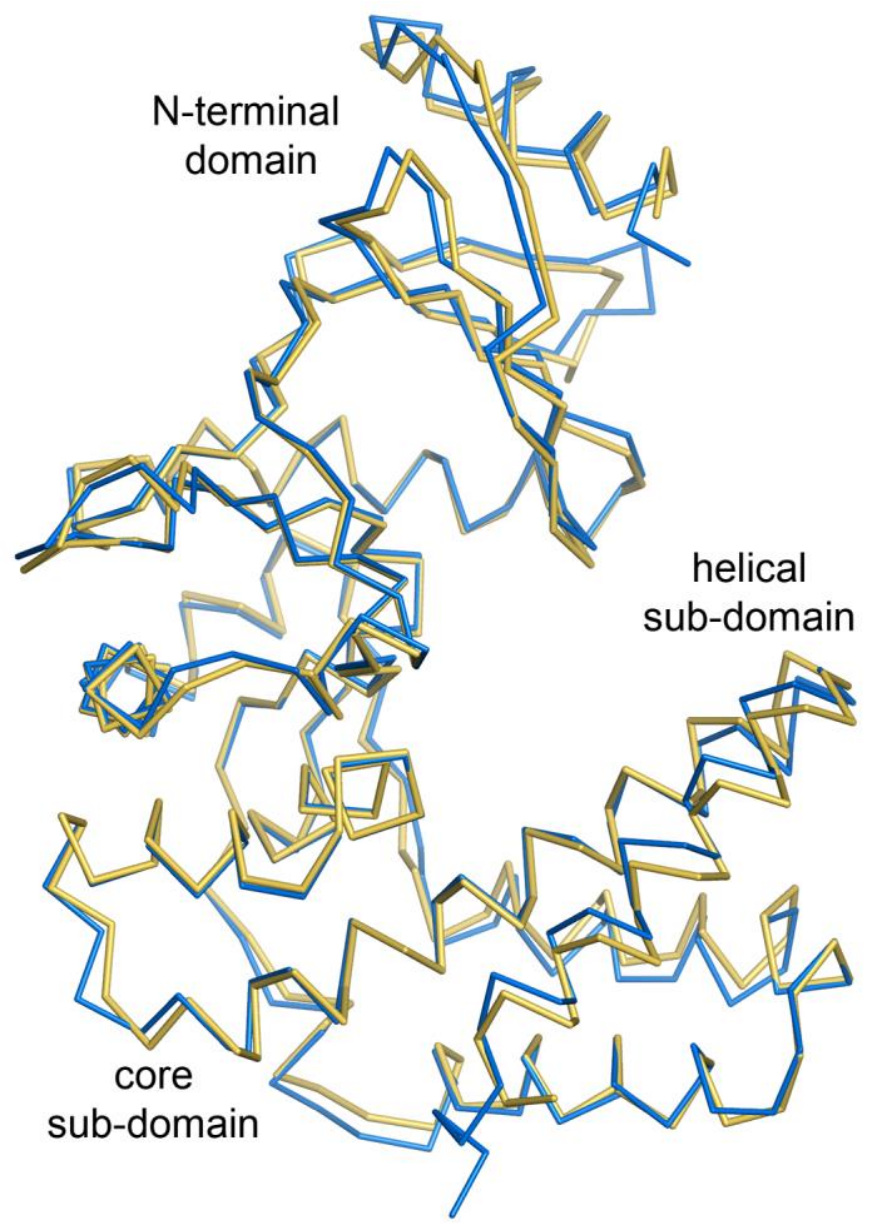

\section{Supplementary Figure S3}

Superposition of the ternary GDP-kanamycin-APH(2")-IIIa complex (yellow) onto the binary GDPAPH(3')-IIIa (PDB code 3TDW, blue), based upon residues in the core sub-domain only. 

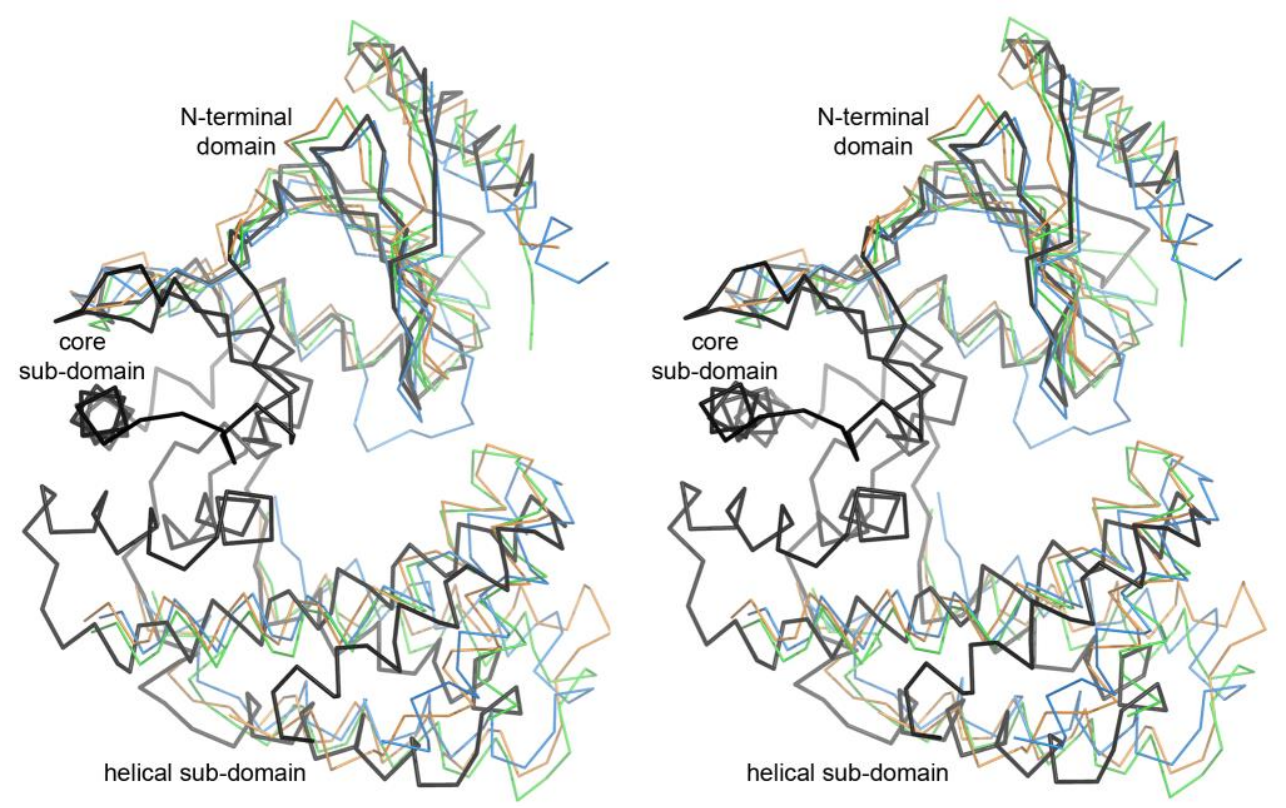

\section{Supplementary Figure S4}

Stereoview of the superposition of APH(2”)-Ia (PDB code 4ORK, blue), APH(2”)-IIa (3HAM, orange) and APH(2")-IVa (3SG9, green) onto APH(2")-IIIa (black), based upon residues in the core subdomain only. For clarity only the core sub-domain of APH(2”)-IIIa is shown. 


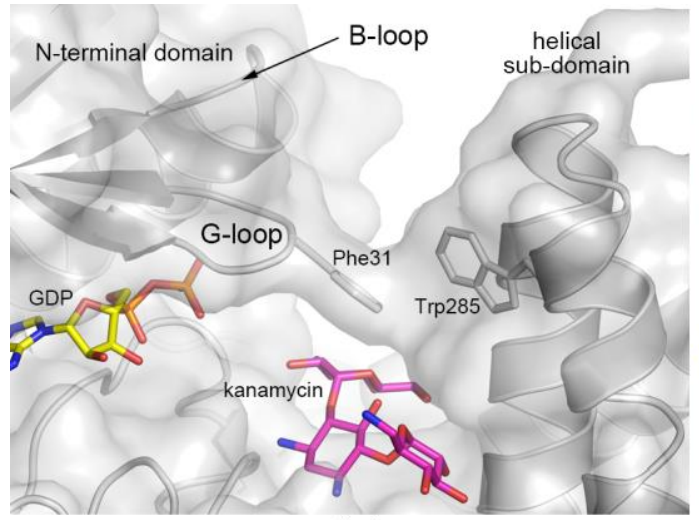

(a)

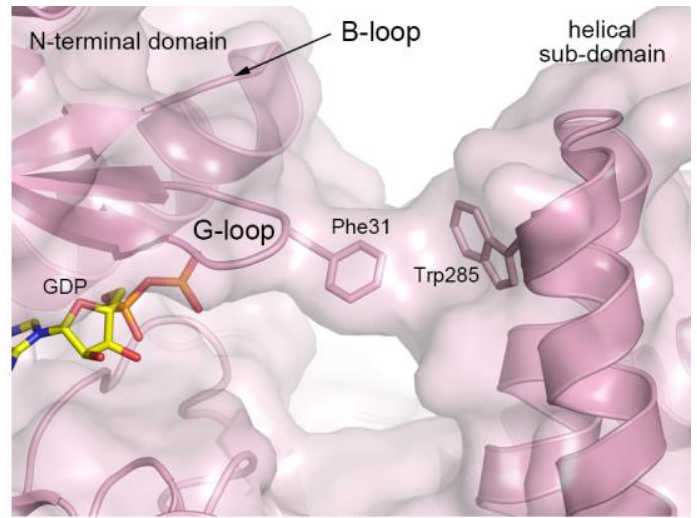

(b)

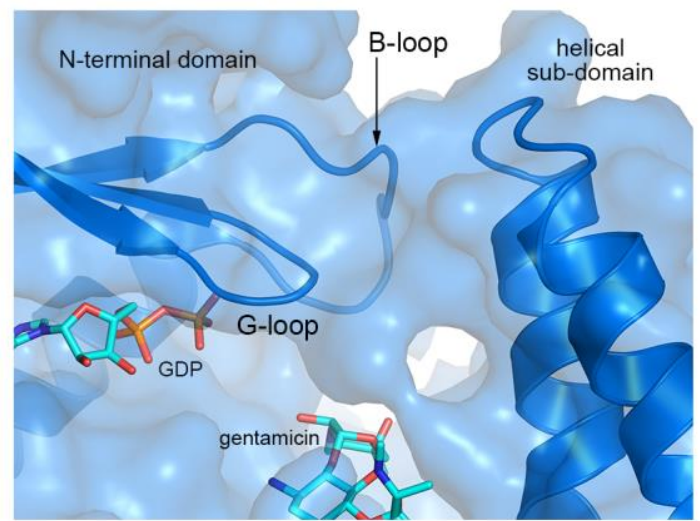

(c)

\section{Supplementary Figure S5}

Solvent accessible surfaces of APH(2")-Ia and APH(2")-IIIa. (a) Semi-transparent surface of ternary kanamycin-GDP-APH(2")-IIIa complex (gray) superimposed onto the ribbon representation. The kanamycin is shown as magenta sticks and the GDP shown as yellow sticks. Only the B- and G-loops from the $\mathrm{N}$-terminal domain (left side), and the $\alpha 9$ and $\alpha 10$ helices from the helical domain (right side) are shown for clarity. (b) Semi-transparent surface of binary GDP-APH(2")-IIIa complex (PDB code 3TDW, light pink) superimposed onto the ribbon representation. The GDP shown as yellow sticks. Only the B- and G-loops from the N-terminal domain (left side), and the $\alpha 9$ and $\alpha 10$ helices from the helical domain (right side) are shown for clarity. (c) Semi-transparent surface of ternary gentamicinGDP-APH(2")-Ia complex (PDB code 5IQG, blue) superimposed onto the ribbon representation. Only the B- and G-loops from the N-terminal domain (left side of the panel), and the $\alpha 9$ and $\alpha 10$ helices from the helical domain (right side) are shown for clarity. The GDP and gentamicin molecules are shown as cyan sticks. 

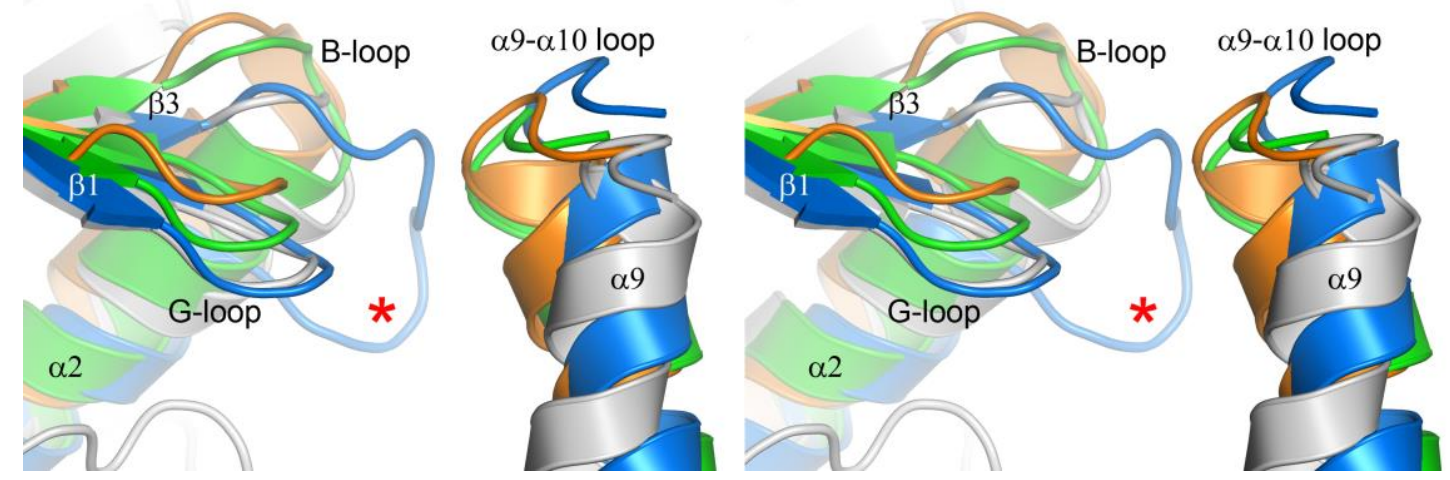

\section{Supplementary Figure S6}

Stereoview of the interface between the N-terminal domain (left) and the helical sub-domain (right) for APH(2")-Ia (PDB code 4ORK, blue), APH(2")-IIa (3HAM, orange), APH(2")-IIIa (gray) and APH(2")-IVa (3SG9, green). The short B-loops in APH(2")-IIa, APH(2")-IIIa and APH(2")-IVa are indicated at the top of the panel, and the extended B-loop for APH(2")-Ia is indicated by a red asterisk. 


\section{Supplementary Table S1}

Hydrogen-bonding interactions between kanamycin and APH(2")-IIIa

\begin{tabular}{|c|c|c|c|c|c|}
\hline kanamycin ring & atom & partner & sub-domain & motif & distance $(\AA)$ \\
\hline \multirow{10}{*}{ A } & O1' (O9) & Glu236 $\mathrm{O}_{\varepsilon 2}$ & core & 2 & 3.16 \\
\hline & O2' (O6) & Glu236 $\mathrm{O}_{\varepsilon 2}$ & core & 2 & 2.94 \\
\hline & & Asp269 $\mathrm{O}_{\delta 2}$ & helical & 3 & 3.01 \\
\hline & & $\mathrm{G} \ln 270 \mathrm{O}_{\varepsilon 1}$ & helical & 3 & 2.59 \\
\hline & & W601 & & $3^{\dagger}$ & 2.70 \\
\hline & O3’ (O7) & $\mathrm{G} \ln 270 \mathrm{~N}_{\varepsilon 2}$ & helical & 3 & 2.94 \\
\hline & & W613 & & & 2.57 \\
\hline & O4’ (O8) & W613 & & & 3.22 \\
\hline & O6' (O5) & W608 & & & 3.28 \\
\hline & N6' (N1) & W611 & & & 3.11 \\
\hline \multirow{8}{*}{ B } & N1 (N3) & Asp196 O & core & 1 & 2.77 \\
\hline & & Ser198 O ${ }_{\gamma}$ & core & 1 & 2.81 \\
\hline & & W610 & & & 2.76 \\
\hline & N3 (N2) & Asp237 $\mathrm{O}_{\delta 1}$ & core & 2 & 3.22 \\
\hline & & Asp237 $\mathrm{O}_{\delta 2}$ & core & 2 & 2.73 \\
\hline & & W605 & & $2^{\dagger}$ & 2.94 \\
\hline & & W611 & & & 2.80 \\
\hline & $\mathrm{O} 5(\mathrm{O} 10)$ & W608 & & & 2.88 \\
\hline \multirow{13}{*}{$\mathrm{C}$} & O2”'(O13) & Asp196 $\mathrm{O}_{\delta 2}$ & core & 1 & 2.74 \\
\hline & & W607 & & & 2.89 \\
\hline & & W606* & & $1^{\dagger}$ & 3.09 \\
\hline & N3” (N4) & Asp221 $\mathrm{O}_{\delta 2}$ & core & 1 & 2.82 \\
\hline & & W603 & & $1^{\dagger}$ & 2.91 \\
\hline & & W604 & & & 2.85 \\
\hline & O4” (O14) & W603 & & $1^{\dagger}$ & 3.20 \\
\hline & & W602 & & & 2.70 \\
\hline & & W609 & & & 2.47 \\
\hline & & W612 & & $3^{\dagger}$ & 3.20 \\
\hline & O6” (O15) & Asp269 $\mathrm{O}_{\delta 1}$ & helical & 3 & 3.25 \\
\hline & & W601 & & $3^{\dagger}$ & $2.06^{\S}$ \\
\hline & & W602 & & & $3.13^{\S}$ \\
\hline
\end{tabular}

$\dagger$ These water molecules make hydrogen-bonding interactions with residues in the three motifs as indicated. "This water molecule is also part of the coordination sphere of one of the magnesium ions bound to the GDP cofactor. \$ The O6" atom is observed in two conformations. In one conformation, O6" forms a hydrogen bond with W602. W601 is also present with this O6" conformer, and may be expelled when O6" adopts the other conformation, since W601 would be too close to form a hydrogen bond in that instance. 


\section{Supplementary Table S2}

Superposition of APH(2")-IIIa onto other APH(2") enzymes ${ }^{\dagger}$

\begin{tabular}{|c|c|c|c|c|}
\hline & & APH(2"')-Ia & APH(2")-IIa & $\mathrm{APH}(2 ")-\mathrm{IVa}$ \\
\hline \multirow[t]{4}{*}{ APH(2")-IIIa } & Overall & $2.8(262)$ & $2.3(249)$ & $2.4(269)$ \\
\hline & N-terminal & $1.5(85)$ & $1.9(84)$ & $1.3(90)$ \\
\hline & core & $1.4(97)$ & $1.9(100)$ & $1.6(100)$ \\
\hline & helical & $2.4(88)$ & $2.8(83)$ & $2.6(81)$ \\
\hline \multirow[t]{4}{*}{ APH(2")-Ia } & Overall & - & $2.1(266)$ & $1.9(270)$ \\
\hline & N-terminal & & $1.7(80)$ & $1.4(88)$ \\
\hline & core & & $1.2(103)$ & $1.1(105)$ \\
\hline & helical & & $2.2(88)$ & $2.0(85)$ \\
\hline \multirow[t]{4}{*}{ APH(2”)-IIa } & Overall & - & - & $1.6(274)$ \\
\hline & N-terminal & & & $1.6(86)$ \\
\hline & core & & & $1.2(103)$ \\
\hline & helical & & & $1.3(84)$ \\
\hline
\end{tabular}

${ }^{\dagger}$ The $r m s d$ s are given in $\AA$, with the number of matching $\mathrm{C} \alpha$ atoms in parentheses. The structures used for the superpositions were PDB code 4ORK (APH(2")-Ia), 3HAM (APH(2”)-IIa) and 3SG9 (APH(2")-IVa. 